\title{
Topography-guided corneal surface laser ablation combined with simultaneous accelerated corneal collagen cross-linking for treatment of keratoconus
}

Yu Zhang and Yueguo Chen ${ }^{*}$

\begin{abstract}
Background: to study the outcomes of topography-guided customized excimer laser subepithelial ablation combined with accelerated CXL for progressive keratoconus.

Methods: Thirty-one eyes of 30 patients with progressive keratoconus were included in this prospective study. Topography-guided excimer laser ablation without refractive correction was performed. Simultaneous accelerated collagen cross-linking with ultraviolet light of $30 \mathrm{~mW} / \mathrm{cm}^{2}$ for 4 min was followed. Uncorrected distance visual acuity (UCVA), manifest refraction, corrected distance visual acuity (CDVA), tomograghy were examined at postoperative 1, 6, and 12 months.

Results: UDVA improved slightly after surgery $(P>0.05)$. BSCDVA improved significantly from $0.32 \pm 0.20$ logMAR to $0.15 \pm 0.14 \log M A R$ at postoperative 12 months $(P<0.05)$. During 12 -month follow-ups, there were no significant differences in manifest refraction and corneal keratometry except for maximal keratometry value of the anterior surface $\left(K_{\text {apex }}\right)$, which decreased significantly from 57.23 $\pm 5.09 \mathrm{D}$ to $53.13 \pm 4.47 \mathrm{D}(P<0.05)$. Even though the thinnest corneal thickness decreased from $465 \pm 24 \mu \mathrm{m}$ to $414 \pm 35 \mu \mathrm{m}(P<0.05)$, curvature asymmetry index front (SIf), keratoconus vertex front (KVf) and Baiocchi Calossi Versaci index front (BCVf) decreased significantly till postoperative 12 months $(P<0.05)$. Corneal higher-order aberrations and coma also decreased significantly till 12 months after surgery $(P<0.05)$.

Conclusions: Topography-guided surface ablation without refractive correction combined with simultaneous accelerated collagen cross-linking provided good stability in refraction and corneal curvature, and also showed significant improvement in BSCDVA, corneal regularity and corneal optical quality.
\end{abstract}

Keywords: Keratoconus, Topography-guided, Laser ablation, Accelerated, Corneal collagen cross-linking

\footnotetext{
* Correspondence: chenyueguo@263.net

Department of Ophthalmology, Beijing key laboratory of restoration of damaged ocular nerve, Peking University Third Hospital, 49 North Huayuan Road, Haidian District, 100191 Beijing, China
}

(c) The Author(s). 2021 Open Access This article is licensed under a Creative Commons Attribution 4.0 International License, which permits use, sharing, adaptation, distribution and reproduction in any medium or format, as long as you give appropriate credit to the original author(s) and the source, provide a link to the Creative Commons licence, and indicate if changes were made. The images or other third party material in this article are included in the article's Creative Commons licence, unless indicated otherwise in a credit line to the material. If material is not included in the article's Creative Commons licence and your intended use is not permitted by statutory regulation or exceeds the permitted use, you will need to obtain permission directly from the copyright holder. To view a copy of this licence, visit http://creativecommons.org/licenses/by/4.0/. The Creative Commons Public Domain Dedication waiver (http://creativecommons.org/publicdomain/zero/1.0/) applies to the data made available in this article, unless otherwise stated in a credit line to the data. 


\section{Background}

Keratoconus is a progressive ectatic corneal disorder that results in corneal stroma impairment and biomechanical weakening. Corneal collagen cross-linking (CXL) is an effective treatment to halt the progression of keratoconus [1]. The classic Dresden CXL uses ultraviolet light of $3 \mathrm{~mW} / \mathrm{cm}^{2}$ illumination and a single treatment process needs $60 \mathrm{~min}$ to reach a total energy of $5.4 \mathrm{~J} / \mathrm{cm}^{2}$ [2]. Recently, researchers have developed accelerated CXL protocols that speed up the procedure using higher-intensity radiation. Several accelerated protocols have been reported to provide comparable results to the classic Dresden CXL [3, 4]. Furthermore, accelerated CXL has been proven to halt the progression of keratoconus in the majority of pediatric patients [5]. Despite reports of well prevention for keratoconus progression and slight improvements of keratometry following CXL, the benefits in terms of improvement in UDVA or CDVA are negligible $[6,7]$. In addition, approximately $15 \%$ of keratoconus patients cannot tolerate contact lenses and some patients have difficulty in fitting the appropriate contact lenses. Thus, some attempts have been made to not only prevent the progression of keratoconus, but also improve visual quality in keratoconus patients.

The combination of CXL and topography-guided photorefractive keratectomy (TG-PRK) was first proposed by Kanellopoulos AJ in 2007, which was known as Athens Protocol $[8,9]$. Previous studies, combining different protocols of CXL and photorefractive treatment performed at the same time or in two-steps, demonstrated a significant improvement of keratometry readings and visual function [9-20]. In these previous studies, however, photorefractive ablation with partial refractive correction was used in all or part of the cases, aiming at reducing not only the lower but also the higher-order aberration and irregular corneal astigmatism [9-20]. As we know, vertical asymmetry and higher-order aberrations are the main reasons for the poor visual function of keratoconus, and lower-order aberrations can be well corrected by spectacles, soft contact lens or implantable contact lens (ICL) implantation. So, photorefractive treatment should focus on correcting only higher-order aberrations, so as to minimize the loss of corneal stroma.

In this prospective study, we examined the evolution of the visual, refractive and tomographic changes during 1-year follow-up after simultaneous TG-PRK without refractive correction followed by accelerated CXL in patients with progressive keratoconus.

\section{Subjects and methods}

\section{Patients}

This prospective study comprised 31 eyes of 30 patients (both eyes of 1 patient), aged between 12 and 34 years (mean, $24.3 \pm 6.3$ years), diagnosed as progressive keratoconus and treated by TG-PRK combined with simultaneous accelerated CXL at Peking University Third Hospital from December, 2016 to March, 2018. KC1 or KC2 was graded on the basis of Amsler and Muckenhirn standard [21]. This study received approval from the ethics committee of Peking University Third Hospital and adhered to the tenets of the Declaration of Helsinki. Written informed consent was obtained from each participant or participant's parents before our interventions.

The inclusion criteria were:(1)Corneal tomography and topography, clinical symptoms and signs demonstrated keratoconus, with a trend of progress in the past 12 months and met one of the following conditions: the maximum $\mathrm{K}$ reading increased by $>1 \mathrm{D}$; mean corneal refractive power increased by $>1 \mathrm{D}$; astigmatism increased by $>1 \mathrm{D}$; the spherical equivalent of manifest refraction increased by $>1.0 \mathrm{D}$ with best spectacle corrected distance visual acuity (BSCDVA) lost more than one line; the thinnest corneal thickness decreased by $>10 \%(2)$. Rigid gas permeable (RGP) contact lens intolerance or inadequate fitting(3)The thinnest thickness of cornea $>450 \mu \mathrm{m}$ and the predicted postoperative thickness of stromal bed $>350 \mu \mathrm{m}$.

The exclusion criteria were:(1)Over mental tension or too young to well cooperate during surgery.(2)Active ocular infection or inflammation, the history of refractive surgery, herpes keratitis, and the ocular diseases other than keratoconus that seriously affected BSCDVA.(3)Prominent corneal scarring and BSCDVA $>1.30$ logMAR.(4)Definitively diagnosed and uncontrolled auto-immune diseases or connective tissue diseases.(5)The women in pregnant or lactating period.

\section{Preoperative Examinations}

All patients had a full ophthalmological examination, including uncorrected distance visual acuity (UDVA), cycloplegic and manifest refractions, BSCDVA, slit-lamp evaluation, Goldmann applanation tonometry, and fundoscopy examinations. With the Sirius combined topographer and tomographer (CSO, Italy), the following parameters were evaluated: maximal keratometry value of the anterior surface $\left(\mathrm{K}_{\text {apex }}\right)$, flat-axis keratometric value (K1), steep-axis keratometric value (K2), corneal astigmatism, minimum corneal thickness (ThkMin), curvature symmetry index front (SIf), keratoconus vertex front (KVf), Baiocchi Calossi Versaci index front (BCVf), root-mean-square of the total higher-order aberrations (HOA-RMS), coma (Coma-RMS), and spherical aberration (SA-RMS). Corneal topography data for topography-guided customized ablation were obtained from the placido-based topographer (Vario Topolyzer, Alcon, USA). Corneal endothelial cell density (ECD), hexagon cell percentage (HEX) and cell area variation 
coefficient (VC) were examined by specular microscopy (Topcon, Japan).

\section{Surgical procedures}

All treatments were performed by the same surgeon (YG Chen). Under topical anesthesia, fresh prepared $20 \%$ alcohol was instilled into the epithelial trephine with a diameter of $9.0 \mathrm{~mm}$, soaking for $20 \mathrm{~s}$. Then the corneal epithelium was peeled and removed. TG-PRK was performed using the Topography-Guided (Topolyzer) software of WaveLight EX500 excimer laser system (Alcon, USA) with an optic zone of $5.0 \sim 6.0 \mathrm{~mm}$. TG-PRK referred to the correction of neither refractive sphere nor cylinder, but $0 \sim-2.25 \mathrm{D}$ of compensated ("measured") cylinder which was automatically calculated based on the Topolyzer topographic results. The ablation depth was calculated automatically by EX500, and controlled within $50 \mu \mathrm{m}$ by reducing the optical zone to some extent. (Fig. 1) Mean maximum ablation depth was $42.16 \pm 2.11 \mu \mathrm{m}(28 \sim 50 \mu \mathrm{m})$. Before and during laser ablation, the static cyclotorsion and kappa angle of the eye were automatically compensated on the basis of the topographic examination. The ablation center was automatically set to the corneal vertex. Mitomycin $\mathrm{C}$ was not used. Irrigation with balanced salt solution was followed by corneal soaking with riboflavin $(0.1 \%$ riboflavin sodium phosphate ophthalmic solution VibeX Rapid; Avedro, Inc) for $10 \mathrm{~min}$. The cornea was irradiated for $4 \mathrm{~min}$ by ultraviolet light $\left(30 \mathrm{~mW} / \mathrm{cm}^{2}, \mathrm{KXL}\right.$ ultraviolet instrument, Avedro, USA) with a total energy delivered of $7.2 \mathrm{~J} / \mathrm{cm}^{2}$. A bandage soft contact lens was applied until complete epithelialization.

\section{Postoperative treatment and follow up}

Levofloxacin $0.5 \%$ eye drops (Santian Pharmaceutical co., LTD, Japan) were applied 4 times a day until epithelial healing. Fluorometholone $0.1 \%$ eye drops (Santian Pharmaceutical co., LTD, Japan) were applied 4 times a day in the first post-operative week and tapered weekly for 4 weeks. After contact lens removal, artificial tears or lubricants were used for at least 1 month according to the conditions of dry eye or delayed healing of epithelium.

The follow-up time points were 1 day, 5 days, 14 days, 1 month, 3 months, 6 months and 12 months after surgery. UDVA and slit lamp examination were performed at every time point. Corneal haze was evaluated according to the system reported by Fantes et al.[22] At 1month, 6-month and 12-month follow-up, manifest refraction, BSCDVA, Sirius combined topographer and tomographer, Topolyzer topographer were examined. At 1-month follow-up, specular microscopy and anterior segment optical coherence tomography (AS-OCT) (Visante OCT; Carl Zeiss Meditec) were examined. Demarcation line depth was measured at corneal vertex using AS-OCT by an experienced technician.

\section{Statistical analysis}

SPSS 21.0 statistical software (SPSS, Inc., Chicago, IL) was used to analyze the data. Continuous data were

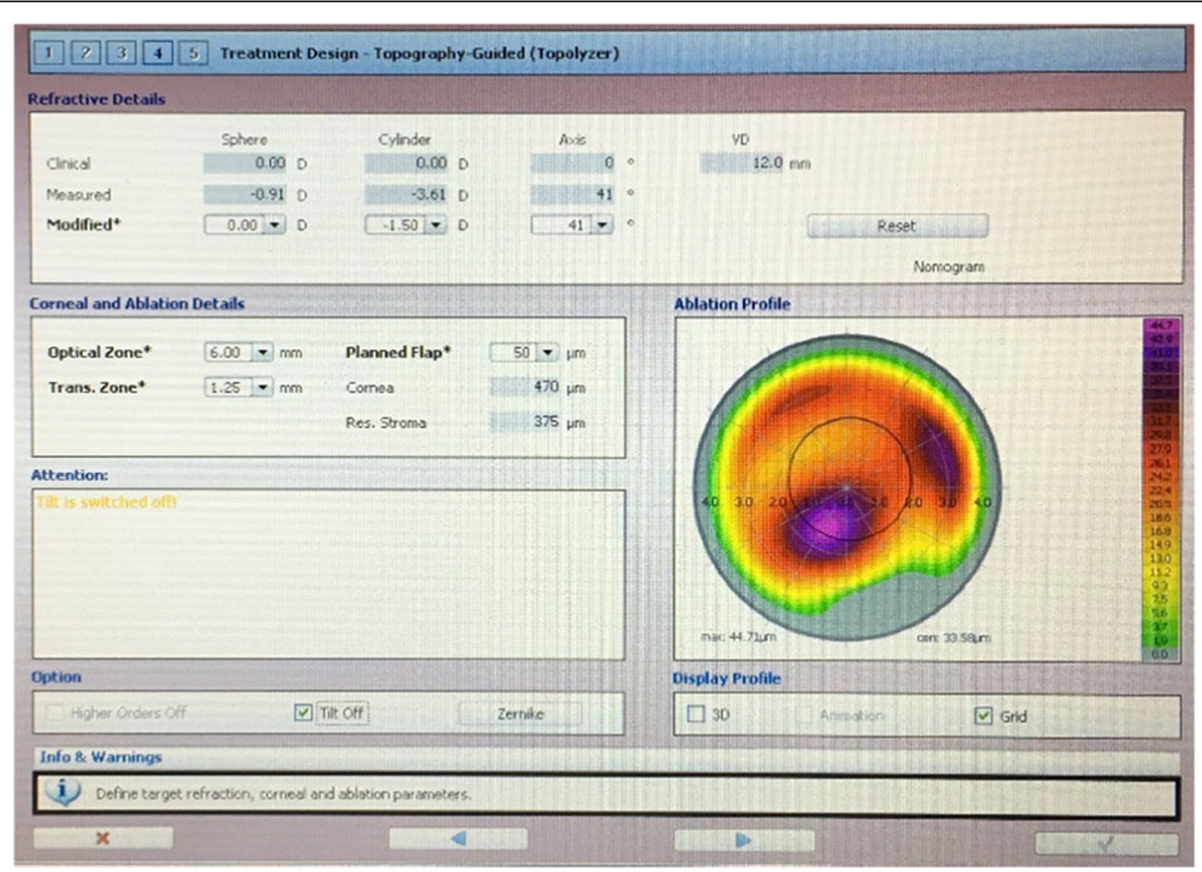

Figure 1 
expressed as mean values \pm standard deviation. Visual acuity was converted to $\log$ MAR for statistical analysis. Repeated measurement variance analysis was used for the comparison of the overall difference of continuous parameters among preoperative and postoperative multiple time points. Dunnett-t test was used for comparing parameters between different two time points. Pearson Chi-Square test was used to compare BSCDVA change between different time points. $P<0.05$ was considered to be statistical significant.

\section{Results}

\section{Visual acuity}

UDVA and BSCDVA detailed data are illustrated in Table 1 . There was no significant difference in UDVA between preoperative and postoperative time points $(P>$ $0.05)$. BSCDVA improved significantly from baseline to postoperative 1 -month and 6-month $(P<0.05)$, and remained relatively stable till postoperative 12 months $(P>0.05)$.

At postoperative 1-month, BSCDVA lost 2 lines in 5 eyes (16\%), 1 line in 1 eye (3\%), unchanged in 6 eyes (19\%) and increased over 2 lines in 16 eyes (52\%). As time went by, BSCDVA improved gradually. At postoperative 12-month, only 1 eye lost 1 line of BSCDVA, and BSCDVA unchanged in 4 eyes $(13 \%)$, and increased over 2 lines in 21 eyes $(68 \%)(P<0.05)$.(Fig. 2$)$

\section{Manifest Refraction}

Table 2 shows the manifest refraction values during follow-ups. There were no significant overall differences in sphere, cylinder and spherical equivalent during the 12 -month follow-up $(P>0.05)$. Only cylinder at postoperative 6 and 12 months decreased significantly, compared to the preoperative value $(P<0.05)$.

\section{Corneal curvature}

Table 3 shows corneal curvature parameters detected by Sirius combined topographer and tomographer during follow-ups. $\mathrm{K}_{\text {apex }}$ decreased significantly at all visits compared to the previous value and the preoperative value $(P<0.05)$. At 12 -month visit, mean $K_{\text {apex }}$ decreased to $53.13 \pm 4.47 \mathrm{D}$, compared to the preoperative mean $\mathrm{K}_{\text {apex }}$ of $57.23 \pm 5.09 \mathrm{D}(P<0.05)$. Although the overall differences in $\mathrm{K} 2$ and mean $\mathrm{K}$ during follow-up were significant, only values at 6 and 12 months were significantly different from 1 -month values $(P<0.05)$. There was no significant difference in corneal cylinder during 12month follow-ups $(P>0.05)$. The corneal topography change of a typical case is shown in Fig. 3.

\section{Keratoconus parameters}

Table 4 shows keratoconus parameters detected by Sirius combined topographer and tomographer during follow-ups. SIf decreased significantly at postoperative 1 month and remained stable until postoperative 12 months $(P<0.001)$. KVf began to decrease significantly at 6 months after operation and continued to decrease till 12 months after operation $(P<0.001)$. BCVf decreased significantly at postoperative 6 months and remained stable until postoperative 12 months $(P<$ 0.001 ). ThkMin decreased significantly at postoperative 1 month, and increased continuously from postoperative 6 months to 12 months $(P<0.001)$.

\section{Corneal aberration}

Corneal HOA and coma decreased significantly at postoperative 1 month, and decreased continuously until 12 months after operation $(P<0.001)$. However, spherical aberration increased significantly at postoperative 1 month, and returned to the preoperative level at postoperative 6 and 12 months $(P<0.05)$. (Table 5$)$

\subsection{Corneal endothelial cell}

There were no significant differences in ECD, HEX and cell area $\mathrm{VC}$ at postoperative 1 month, compared to the preoperative values $(P>0.05)$. (Table 6$)$

\section{Postoperative corneal changes and complications}

At postoperative 1 month, the demarcation line was well defined in each case with a mean depth of $216 \pm 25 \mu \mathrm{m}$. Aseptic inflammatory sub-epithelial infiltration occurred in one eye (3.2\%). No extra specific treatment was used and the opacity was basically absorbed within 4 weeks. At postoperative 1 month, corneal haze started to appear and dissipated gradually from postoperative 3 months to 12 months. The number of eyes with haze of grade 0 , $0.5,1$, and 2 at postoperative 12-month follow-up were 25 eyes (80.6\%), 4 eyes (13.0\%), 1 eye (3.2\%), and 1 eye (3.2\%), respectively.

Table 1 Mean changes in visual acuity over 12- month follow-up $(n=31, x \pm s)$

\begin{tabular}{|c|c|c|c|c|c|c|}
\hline & Preoperative & 1-month & 6-month & 12-month & $F$ & $P$ \\
\hline UDVA(LogMAR) & $0.79 \pm 0.33$ & $0.76 \pm 0.23$ & $0.70 \pm 0.27$ & $0.71 \pm 0.27$ & 2.016 & 0.134 \\
\hline BSCDVA(LogMAR) & $0.32 \pm 0.20$ & $0.24 \pm 0.24^{*}$ & $0.16 \pm 0.22^{* \#}$ & $0.15 \pm 0.14^{* \#}$ & 13.495 & $<0.001$ \\
\hline
\end{tabular}

Abbreviations: UDVA uncorrected distance visual acuity; BSCDVA best spectacle corrected distance visual acuity

* significant difference compared with preoperative baseline $(P<0.05)$

\# significant difference compared with postoperative 1-month $(P<0.05)$ 


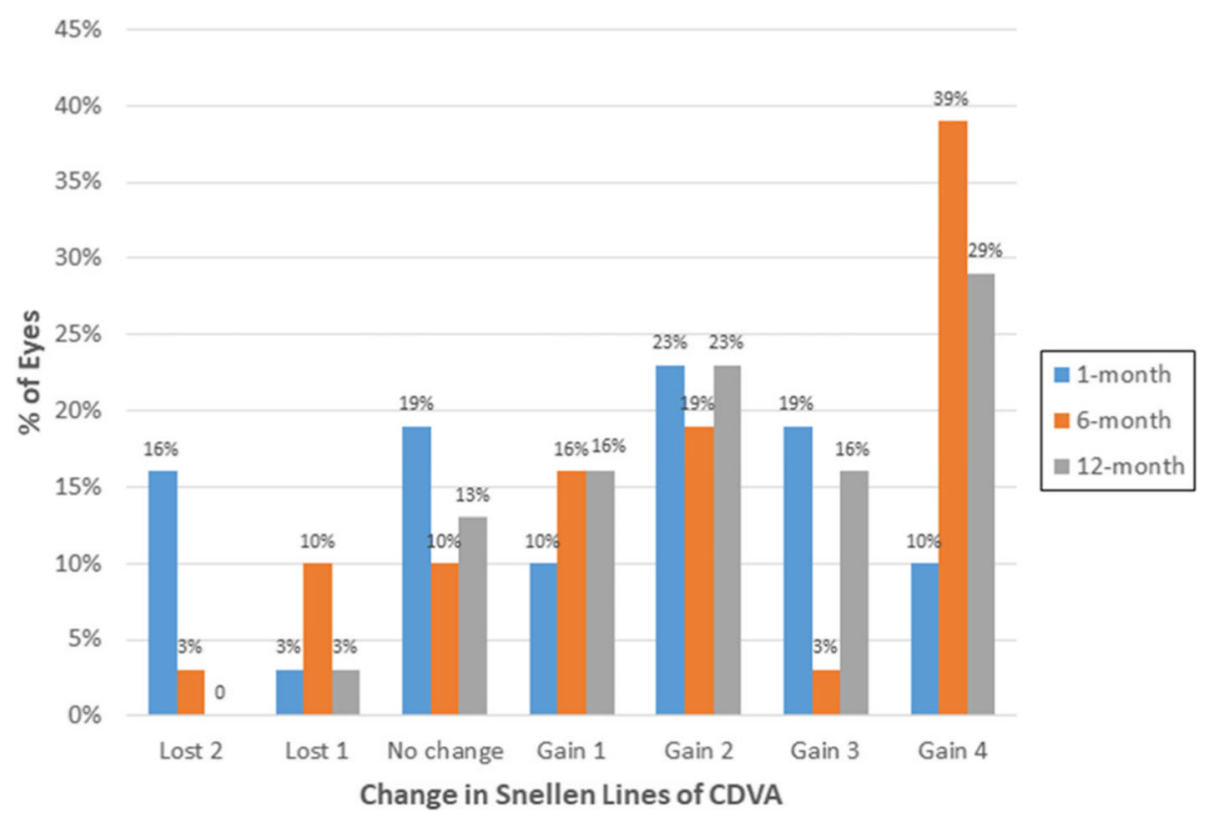

Figure 2

\section{Discussion}

The classic Dresden CXL using ultraviolet light of 3mw/ $\mathrm{cm}^{2}$ illumination is a time-consuming procedure, which can cause high percentage of haze [19] and the risk of progressive corneal flattening [20], especially in simultaneous combined treatments. The accelerated CXL uses ultraviolet light of high irradiation intensity. According to the Bunsen-Roscoe law of photochemical effect, the higher the illumination, the shorter the exposure time [23].The exposure time required is greatly shortened, which improves the treatment efficiency and increases patients' compliance. Additionally, accelerated CXL was proved to be effective and have fewer complications [4, 5]. So, recently, accelerated CXL has been widely used by most surgeons for CXL combined treatment [13, 16$18,20]$. In the current study, $30 \mathrm{~mW} / \mathrm{cm}^{2}$ ultraviolet light intensity of illumination for $4 \mathrm{~min}$ was used for the first time.

Initially, epithelial removal prior to CXL was performed using manual debridement (with or without alcohol) [13-15]. Recently, transepithelial phototherapeutic keratectomy (PTK) has also been employed as a method for removing the epithelial [16-
18]. Because of a doughnut-shaped model of the corneal epithelium in keratoconus [24], PTK removes some stromal tissue from the central cone, which flattens the cornea more than manual debridement [25], but consumes more corneal stromal tissue at the cone apex. The corneal surface following PTK is more consistent with the preoperative topography, which makes the subsequent TG-PRK become more accurate. PTK epithelial removal leaves some epithelium around the cone, which may reduce the ablation volume of the corneal stromal tissue during TG-PRK, but can cause under-correction. In the present study, manual debridement with alcohol was used, which could reduce the loss of corneal stroma at the cone apex to the greatest extent, and reduced the excessive friction stimulation of corneal stroma by mechanical scraping.

Topography-guided customized ablation attempts to maintain the aspheric shape of the cornea and neutralize corneal irregularities [26]. It has been shown to be effective in treating irregular astigmatism caused by iatrogenic corneal irregularities[27]. Since topography-guided ablation for normalizing the anterior cornea can bring in refractive change, especially astigmatism change [28], the

Table 2 Mean changes in manifest refraction over 12-month follow-up. ( $n=31, x \pm s)$

\begin{tabular}{llllll}
\hline & Preoperative & 1-month & 6-month & 12-month & F \\
\hline Sphere(D) & $-5.56 \pm 4.14$ & $-5.16 \pm 4.09$ & $-5.30 \pm 4.44$ & $-5.19 \pm 4.35$ & 0.478 \\
Cylinder(D) & $-3.75 \pm 1.53$ & $-3.43 \pm 2.12$ & $-3.21 \pm 1.85^{*}$ & $-3.07 \pm 1.96^{*}$ & 0.700 \\
SE(D) & $-7.43 \pm 4.52$ & $-6.87 \pm 4.29$ & $-6.92 \pm 4.78$ & $-6.65 \pm 4.67$ & 1.810 \\
\hline
\end{tabular}

Abbreviations: SE spherical equivalent

* significant difference compared with preoperative baseline $(P<0.05)$ 
Table 3 Mean changes in corneal curvature parameters over 12-month follow-up $(n=31, x \pm s)$

\begin{tabular}{|c|c|c|c|c|c|c|}
\hline & Preoperative & 1-month & 6-month & 12-month & $F$ & $P$ \\
\hline $\begin{array}{l}K_{\text {apex }}(D) \\
\text { K1(D) } \\
\text { K2(D) } \\
\text { mean K(D) } \\
\text { CYL(D) }\end{array}$ & $\begin{array}{l}57.23 \pm 5.09 \\
45.47 \pm 2.35 \\
48.35 \pm 2.94 \\
46.86 \pm 2.53 \\
-2.87 \pm 1.54\end{array}$ & $\begin{array}{l}54.96 \pm 4.44^{*} \\
45.64 \pm 2.71 \\
48.73 \pm 3.47 \\
47.12 \pm 2.97 \\
-3.09 \pm 1.74\end{array}$ & $\begin{array}{l}54.24 \pm 4.87^{*} \\
45.31 \pm 2.83^{\#} \\
48.23 \pm 3.26^{\#} \\
46.69 \pm 2.98^{\#} \\
-2.91 \pm 1.61\end{array}$ & $\begin{array}{l}53.13 \pm 4.47^{* \# \&} \\
45.28 \pm 2.77^{\#} \\
48.09 \pm 3.04^{\#} \\
46.63 \pm 2.79^{\#} \\
-2.79 \pm 1.60\end{array}$ & $\begin{array}{l}29.453 \\
1.794 \\
4.077 \\
3.775 \\
1.707\end{array}$ & $\begin{array}{l}<0.001 \\
0.171 \\
0.016 \\
0.022 \\
0.188\end{array}$ \\
\hline
\end{tabular}

Abbreviations: $K_{\text {apex }}$ maximal keratometry value of the front surface; $K$ flat-axis keratometric value; $K 2$ steep-axis keratometric value; $M e a n ~ K$ mean keratometric value; $C Y L$ corneal cylinder

* significant difference compared with preoperative baseline $(P<0.05)$

\# significant difference compared with postoperative 1 -month $(P<0.05)$

\& significant difference compared with postoperative 6-month $(P<0.05)$

clinical refraction should be adjusted to keep it neutral after refractive correction. Kanellopoulos found that topography-modified refraction (TMR) offered superior refractive and visual outcomes to standard clinical refraction in myopic topography-guided LASIK [29]. In the current study, we used TMR for reference to compensate for partial cylinder measured by topographer without refractive correction while topography-guided ablation of irregular corneas on the basis of ensuring the depth of corneal ablation.

In the current study, UDVA improved slightly after surgery, but there was no statistical significance. BSCDVA improved significantly from $0.32 \pm 0.20$ logMAR to $0.15 \pm 0.14 \log$ MAR at postoperative 12 months $(P<0.05)$. Manifest refraction, flat $\mathrm{K}$, steep $\mathrm{K}$ and corneal cylinder all decreased slightly after surgery, but no statistical significance was found. However, $K_{\text {apex }}$ flattened significantly after surgery. Generally speaking, the improvement of UDVA, the decrease of refraction and corneal curvature were not obvious, which were different from most previous studies [10-20]. Because of partial correction of clinical refraction, there were improvements in UDVA [8-13, 15-18], curvature readings [13, $15,17-20]$ and manifest refraction $[13,15,16,18-20]$ after surgery. The CXL plus TG-PRK in the present study aimed at halting the progression of keratoconus and reducing corneal HOA, so we did not carry out refractive correction, which was a main difference between the present study and previous studies [10-20]. Despite of the different TG-PRK protocol, the present study still showed improvements in BSCDVA and $\mathrm{K}_{\mathrm{apex}}$ similar to the previous studies [10-20], which increased the

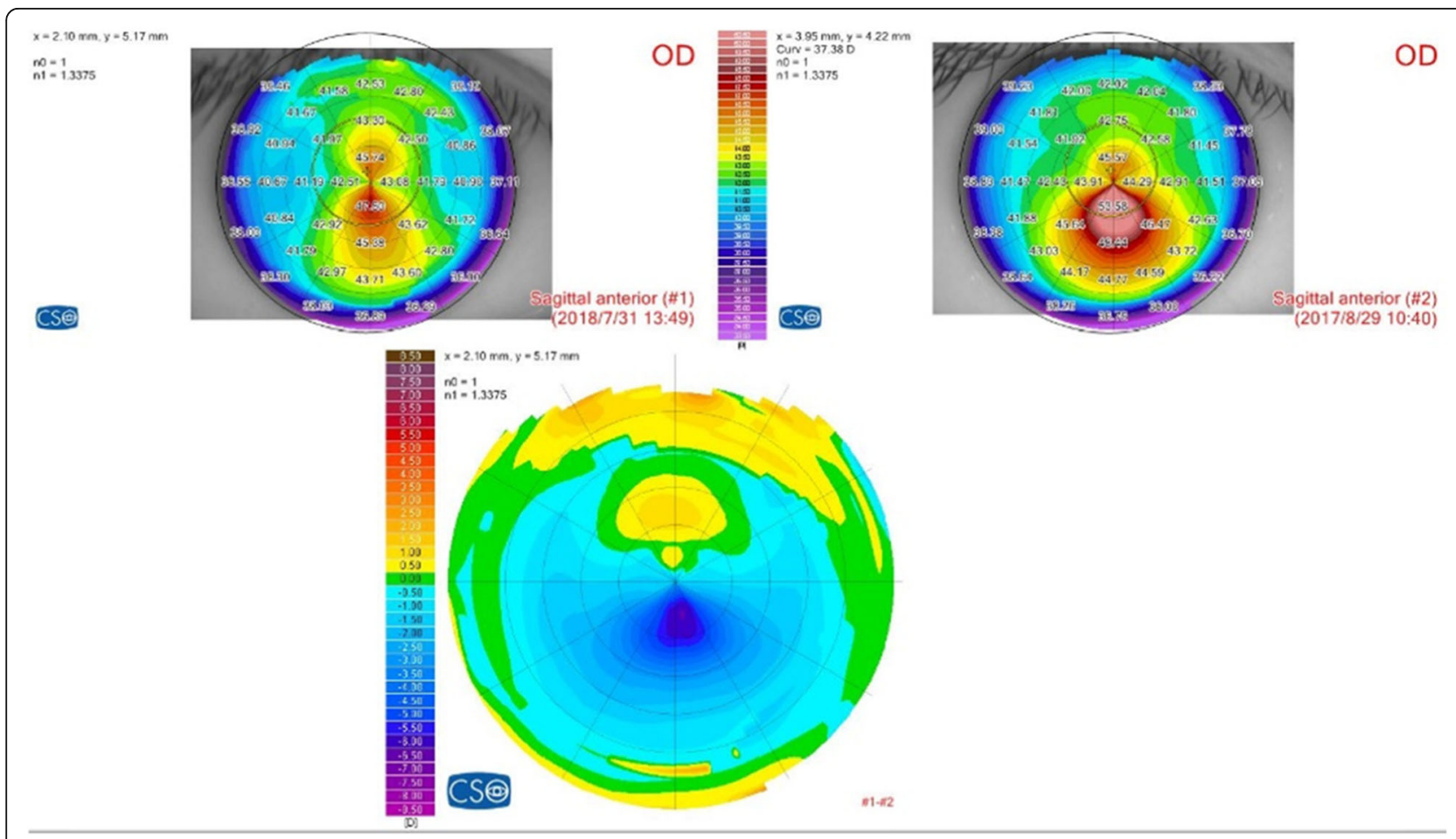

Figure 3 
Table 4 Mean changes in keratoconus parameters over 12-month follow-up ( $n=31, x \pm s)$

\begin{tabular}{lllllll}
\hline & Preoperative & 1-month & 6-month & 12-month & F & P \\
\hline SIf $(\mathrm{D})$ & $5.65 \pm 3.12$ & $3.64 \pm 3.20^{*}$ & $3.62 \pm 2.66^{*}$ & $3.30 \pm 2.82^{*}$ & 21.950 & $<0.001$ \\
KVf $(\mu \mathrm{m})$ & $30.32 \pm 12.62$ & $31.00 \pm 13.89$ & $27.97 \pm 12.66^{* \#}$ & $26.77 \pm 12.23^{* \# \&}$ & 19.741 & $<0.001$ \\
BCVf $(\mu \mathrm{m})$ & $2.93 \pm 1.29$ & $2.88 \pm 1.48$ & $2.47 \pm 1.48^{* \#}$ & $2.49 \pm 1.25^{* \#}$ & 8.357 & $<0.001$ \\
ThkMin $(\mu \mathrm{m})$ & $465 \pm 24$ & $387 \pm 34^{*}$ & $400 \pm 32^{* \#}$ & $414 \pm 35^{* \# \&}$ & 75.081 & $<0.001$ \\
\hline
\end{tabular}

Abbreviations: SIf curvature symmetry index front; KVf keratoconus vertex front; BCV Baiocchi Calossi Versaci index front; ThkMin thinnest corneal thickness * significant difference compared with preoperative baseline $(P<0.05)$

\# significant difference compared with postoperative 1-month $(P<0.05)$

\& significant difference compared with postoperative 6-month $(P<0.05)$

correction effect of frame glasses or ICL implantation when contact lenses were not tolerated or helped to regain a good contact lenses fitting.

The current study also showed that SIf, KVf and BCVf, which reflected the irregularity of the anterior corneal surface, reduced significantly after surgery and remained in a reduced state up to 12 months after surgery. Those indicated that the cornea became regular after surgery and resulted in the improvement of BSCDVA. Some previous studies showed similar results that index of surface variance (ISV) and index of height decentration (IHD) decreased significantly after surgery[17]. The current study also found that corneal aberrations including corneal HOA and coma decreased continuously within 12 months after surgery, which also indicated the improvement of corneal regularity due to TG ablation. Ahmet et al.[16] and Rechichi et al. [18] found that corneal HOA, coma and spherical aberration (SA) all significantly decreased at 24 months after surgery. The difference of the change of corneal SA might related to the different excimer laser system (Schwind Amaris, Germany) and different ablation mode (Transepithelial TG-PRK).

Like some previous studies $[10,12,16,18]$, MMC was not used in the present study. Kymionis GD et al. found that CXL could destroy the regeneration of corneal anterior stromal cells by confocal microscopy, so it was not necessary to use MMC after PRK combined CXL [30]. The synergistic effect of CXL and MMC may cause more cell death and more corneal haze [19].Although haze was observed at each case in postoperative one month in the present study, it gradually faded away 3 to 12 months after operation. This slightly obvious haze may be related to alcohol deepithelialization and 4-week treatment of glucocorticoid eyedrops after surgery.

The limitations of this study are as follows. First, this study had a small sample and was lack of a control group. Second, we didn't estimate the impact of corneal posterior surface when planning TG ablation. In future, software based on calculation of mean pupillary power or raytracing technology compensating anterior and posterior corneal surfaces refractive and aberrometric contributions are mandatory to optimize visual and refractive outcomes. Third, like most previous studies, we used uniform protocol in all cases. Pachymetry-based or topography-guided customized CXL might be more effective and safer [18, 31, 32]. Rechichi et al.[18]offset ablation center towards the location of cone apex, which might better reduce coma. These customized procedures provided a good reference for our surgical protocol in the future.

It has been reported that a small number of keratoconus (around $8 \%$ ) may still progress after CXL [33]. Thus regular and long-term follow-up of refraction and corneal topography/tomography at different time points after surgery is necessary, especially after the combined surgery with reduced corneal thickness. The research of long-term safety is warrant.

\section{Conclusions}

In conclusion, the current study showed the significant improvement in BSCDVA, $K_{\text {apex }}$, corneal irregularity indices and RMS of HOA and coma, and also showed good stability in refraction and corneal curvature after simultaneous TG-PRK without refractive correction followed by accelerated CXL $\left(30 \mathrm{~mW} / \mathrm{cm}^{2}\right)$ in patients

Table 5 Mean changes in corneal aberration parameters over 12-month follow-up $(n=31, x \pm s)$

\begin{tabular}{llllll}
\hline & Preoperative & 1-month & 6-month & 12-month & F \\
\hline HOA-RMS & $2.25 \pm 0.84$ & $2.02 \pm 0.98^{*}$ & $1.71 \pm 0.85^{* \#}$ & $1.57 \pm 0.85^{* \# \&}$ & 32.796 \\
Coma-RMS & $1.82 \pm 0.78$ & $1.46 \pm 0.93^{*}$ & $1.26 \pm 0.81^{* \#}$ & $1.14 \pm 0.79^{* \# \&}$ & 21.115 \\
SA-RMS & $0.45 \pm 0.36$ & $0.78 \pm 0.55^{*}$ & $0.54 \pm 0.40^{\#}$ & $0.54 \pm 0.39^{\#}$ & $<.001$ \\
\hline
\end{tabular}

Abbreviations: HOA-RMS root-mean-square of the total higher-order aberrations; Coma-RMS root-mean-square of coma; SA-RMS root-mean-square of spherical aberration

*: significant difference compared with preoperative baseline $(P<0.05)$

\#: significant difference compared with postoperative 1-month $(P<0.05)$

\&: significant difference compared with postoperative 6-month $(P<0.05)$ 
Table 6 Mean changes in corneal endothelial cell parameters at 1-month follow-up $(n=31, x \pm s)$

\begin{tabular}{lllll}
\hline & Preoperative & 1-month & t & $\boldsymbol{P}$ \\
\hline ECD & $2953.24 \pm 120.38$ & $2994.81 \pm 72.51$ & -0.493 & 0.633 \\
HEX(\%) & $61.64 \pm 5.82$ & $54.00 \pm 3.96$ & 1.279 & 0.230 \\
VC (\%) & $33.41 \pm 1.60$ & $33.49 \pm 1.94$ & -0.065 & 0.949 \\
\hline
\end{tabular}

Abbreviations: $E C D$ endothelial cell density; HEX hexagon cell percentage;

$V C$ cell area variation coefficient

with progressive mild-to-moderate keratoconus. The treatment protocol is convenient, time-saving, effective and safe. However, large-scale, comparative, long-term trials are required to determine the optimum parameters and evaluate the long-term safety and effectiveness of this combined surgery.

\begin{abstract}
Abbreviations
CXL: Corneal collagen cross-linking; UCVA: Uncorrected distance visual acuity; CDVA: Corrected distance visual acuity; SIf: Curvature symmetry index front; KVf: Keratoconus vertex front; BCVf: Baiocchi Calossi Versaci index front; TGPRK: Topography-guided photorefractive keratectomy;

PTK: Phototherapeutic keratectomy; BSCDVA: Best spectacle corrected distance visual acuity; $\mathrm{K}_{\text {apex }}$ : Maximal keratometry value of the anterior surface; K1: Flat-axis keratometric value; K2: Steep-axis keratometric value; ThkMin: Minimum corneal thickness; HOA-RMS: Root-mean-square of the total higher-order aberrations; Coma-RMS: Root-mean-square of coma; SARMS: Root-mean-square of spherical aberration; ECD: Corneal endothelial cell density; HEX: Hexagon cell percentage; VC: variation coefficient; ASOCT: Anterior segment optical coherence tomography; ICL: Implantable contact lens
\end{abstract}

\section{Acknowledgements}

Not applicable.

\section{Authors' contributions}

YZ analyzed and interpreted the patient data, and wrote the manuscript. YC designed the study, collected data and revised the manuscript. All authors read and approved the final manuscript.

\section{Funding}

Key Clinical Innovation Program of Peking University third hospital, category A (BYSYZD2019002).

Key Clinical Innovation Program of Peking University third hospital, category B (BYSY2018027).

\section{Availability of data and materials}

The datasets generated and/or analysed during the current study are not publicly available due to limitations of ethical approval involving the patient data and anonymity but are available from the corresponding author on reasonable request.

\section{Declarations}

\section{Ethics approval and consent to participate}

This study was carried out in accordance with the tenets of the Declaration of Helsinki. It was approved by the Ethics Committee of Peking University Third Hospital. An informed consent was obtained from each subject.

\section{Consent for publication}

Not applicable.

\section{Competing interests}

The authors declare that they have no competing interests.
Received: 13 March 2021 Accepted: 9 July 2021

Published online: 24 July 2021

\section{References}

1. Raiskup F, Theuring A, Pillunat LE, Spoerl E. Corneal collagen crosslinking with riboflavin and ultraviolet-a light in progressive keratoconus: ten-year results. J Cataract Refract Surg 2015; 41(1): 41-46.

2. Wollensak G. Crosslinking treatment of progressive keratoconus: new hope. Curr Opin Ophthalmol 2006; 17(4): 356-360.

3. Hagem AM, Thorsrud A, Sandvik GF, Drolsum L. Randomized study of collagen cross-linking with conventional versus accelerated UVA irradiation using riboflavin with hydroxypropyl methylcellulose: two-year results. Cornea 2019; 38(2):203-209.

4. Males JJ, Viswanathan D. Comparative study of long-term outcomes of accelerated and conventional collagen crosslinking for progressive keratoconus. Eye (Lond) 2018; 32(1):32-38.

5. Ağca A, Tülü B, Yasa D, et al. Accelerated corneal crosslinking in children with keratoconus: 5-year results and comparison of 2 protocols. J Cataract Refract Surg 2020; 46(4):517-523.

6. Zarei-Ghanavati S, Khakshour H, Vejdani M, Ghooshkhanei H, Vejdani A. Evaluation of changes in visual acuity, contrast sensitivity and aberrations in patients with keratoconus after corneal collagen cross-linking. J Ophthalmic Vis Res 2017;12(3):260-264

7. Uysal BS, Sarac O, Yaman D, Akcay E, Cagil N. Optical performance of the cornea one year following keratoconus treatment with corneal collagen cross-linking. Curr Eye Res 2018; 43(12): 1415-1421.

8. Kanellopoulos AJ, Binder PS. Collagen cross-linking (CCL) with sequential topography-guided PRK: a temporizing alternative for keratoconus to penetrating keratoplasty. Cornea. 2007;26(7):891-895.

9. Kanellopoulos AJ, Binder PS. Management of corneal ectasia after LASIK with combined, same-day, topography-guided partial transepithelial PRK and collagen cross-linking: the athens protocol. J Refract Surg. 2011;27(5): 323-331.

10. Kymionis GD, Kontadakis GA, Kounis GA, Portaliou DM, Karavitaki AE, Magarakis M, Yoo S, Pallikaris IG. Simultaneous topography-guided PRK followed by corneal collagen cross-linking for keratoconus. J Refract Surg. 2009 Sep;25(9): S807-11.

11. Kanellopoulos AJ. The management of cornea blindness from severe corneal scarring, with the Athens Protocol (transepithelial topographyguided PRK therapeutic remodeling, combined with same-day, collagen cross-linking). Clin Ophthalmol. 2012;6:87-90.

12. Kymionis GD, Grentzelos MA, Kankariya VP, Pallikaris IG. Combined transepithelial phototherapeutic keratectomy and corneal collagen crosslinking for ectatic disorders: cretan protocol. J Cataract Refract Surg. 2013 Dec;39(12):1939.

13. Sakla H, Altroudi W, Munoz G, Sakla Y. Simultaneous Topography-Guided Photorefractive Keratectomy and Accelerated Corneal Collagen CrossLinking for Keratoconus. Cornea 2016; 35(7): 941-945.

14. Kanellopoulos AJ. Comparison of sequential vs same-day simultaneous collagen cross-linking and topography-guided PRK for treatment of keratoconus. J Refract Surg 2009; 25(9): S812-818.

15. Sakla H, Altroudi W, Muñoz G, Albarrán-Diego C. Simultaneous topographyguided partial photorefractive keratectomy and corneal collagen crosslinking for keratoconus. J Cataract Refract Surg 2014; 40(9): 1430-1438.

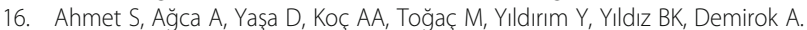
Simultaneous transepithelial topography-guided photorefractive keratectomy and accelerated cross-linking in keratoconus: 2-year follow-up. Biomed Res Int 2018: 2945751.

17. Kanellopoulos AJ, Vingopoulos F, Sideri AM. Long-term stability with the Athens protocol (topography-guided partial PRK combined with crosslinking) in pediatric patients with keratoconus. Cornea 2019; 38(8): 10491057

18. Rechichi M, Mazzotta C, Oliverio GW, Romano V, Borroni D, Ferrise M, Bagaglia S, Jacob S, Meduri A. Selective transepithelial ablation with simultaneous accelerated Corneal Cross-linking for corneal regularization of keratoconus: the STARE-X Protocol. J Cataract Refract Surg. 2021 Mar 9. Epub ahead of print.

19. Renata L B Moraes, Ramon C Ghanem, Vinicius C Ghanem, Marcony R Santhiago. Haze and Visual Acuity Loss After Sequential Photorefractive Keratectomy and Corneal Cross-linking for Keratoconus. J Refract Surg. 2019; 35(2):109-14. 
20. Kanellopoulos AJ. Ten-Year Outcomes of Progressive Keratoconus Management With the Athens Protocol (Topography-Guided PartialRefraction PRK Combined With CXL). J Refract Surg. 2019;35(8):478-483.

21. Kamiya K, Ishii R, Shimizu K, Igarashi A. Evaluation of corneal elevation, pachymetry and keratometry in keratoconic eyes with respect to the stage of Amsler-Krumeich classification. Br J Ophthalmol 2014; 98(4):459-463.

22. Fantes FE, Hanna KD, Waring GO III, Pouliquen Y, Thompson KP, Savoldelli M. Wound healing after excimer laser keratomileusis (photorefractive keratectomy) in monkeys. Arch Ophthalmol 1990;108(5):665-675.

23. Wernli J, Schumacher S, Spoerl E, et al. The efficacy of corneal cross-linking shows a sudden decrease with very high intensity UV light and short treatment time. Invest Ophthalmol Vis Sci 2013, 54(2): 1176-1180.

24. Reinstein DZ, Gobbe M, Archer TJ, Silverman RH, Coleman DJ. Epithelial, stromal, and total corneal thickness in keratoconus: three-dimensional display with Artemis very-high frequency digital ultrasound. J Refract Surg 2010; 26:259-271.

25. Gaster RN, Ben Margines J, Gaster DN, Li X, Rabinowitz YS. Comparison of the Effect of Epithelial Removal by Transepithelial Phototherapeutic Keratectomy or Manual Debridement on Cross-linking Procedures for Progressive Keratoconus. J Refract Surg. 2016;32(10):699-704.

26. Pasquali T, Krueger R. Topography-guided laser refractive surgery. Curr Opin Ophthalmol 2012; 23(4): 264-268.

27. Ghoreishi M, Naderi Beni A, Naderi Beni Z. Visual outcomes of topographyguided excimer laser surgery for treatment of patients with irregular astigmatism. Lasers Med Sci 2014; 29(1):105-111.

28. Motwani M. The use of WaveLight ${ }^{\oplus}$ Contoura to create a uniform cornea: the LYRA Protocol. Part 1: the effect of higher-order corneal aberrations on refractive astigmatism. Clin Ophthalmol 2017;16(11):897-905.

29. Kanellopoulos AJ. Topography-modified refraction(TMR): adjustment of treated cylinder amount and axis to the topography versus standard clinical refraction in myopic topography-guided LASIK. Clinical Ophthalmology 2016;3(10):2213-2221.

30. Kymionis GD, Diakonis VF, Kalyvianaki M, et al. One-year follow-up of corneal confocal microscopy after corneal crosslinking in patients with post laser in situ keratosmileusis ectasia and keratoconus. Am J Ophthalmol. 2009; 147(5):774-778.

31. Mazzotta C, Romani A, Burroni A. Pachymetry-based accelerated crosslinking: the "M Nomogram" for standardized treatment of all-thickness progressive ectatic corneas. Int J Keratoconus Ectatic Corneal Dis. 2019;7(2): $137-44$.

32. Kanellopoulos AJ. Management of progressive keratoconus with partial topography-guided PRK combined with refractive, customized CXL- a novel technique: the enhanced Athens protocol. Clinical Ophthalmology 2019;13: 581-588.

33. Shalchi Z, Wang $X$, Nanavaty MA. Safety and efficacy of epithelium removal and transepithelial corneal collagen crosslinking for keratoconus. Eye 2015; 29(1): 15-29.

\section{Publisher's Note}

Springer Nature remains neutral with regard to jurisdictional claims in published maps and institutional affiliations.

Ready to submit your research? Choose BMC and benefit from:

- fast, convenient online submission

- thorough peer review by experienced researchers in your field

- rapid publication on acceptance

- support for research data, including large and complex data types

- gold Open Access which fosters wider collaboration and increased citations

- maximum visibility for your research: over $100 \mathrm{M}$ website views per year

At $\mathrm{BMC}$, research is always in progress.

Learn more biomedcentral.com/submissions 